\title{
Matthias Jarke
}

\section{Transkriptivität als informatisches Designprinzip}

Das Aachen-Bonn-Kölner Kulturwissenschaftliche Forschungskolleg »Medien und Kulturelle Kommunikation « (SFB/FK 427) ist nach einem Rahmenwerk organisiert, welches das Arbeiten mit Medien mit den Schlagworten »Transkribieren - Adressieren - Lokalisieren« charakterisiert. Dieses von seinem Initiator Ludwig Jäger zunächst deskriptiv gemeinte Rahmenwerk wird hier als informatisches Konstruktionsprinzip aufgefasst.

Nach einem kurzen Überblick über den Gesamtansatz werden vergleichend einige Beobachtungen zum Medieneinsatz in kulturwissenschaftlichen und ingenieurwissenschaftlichen Projekten unter diesem Blickwinkel gewertet (vgl. S. 130 ff.). Aus diesen Beobachtungen wurde eine Architektur für kooperative internet-basierte Transkriptions-, Lokalisierungs- und Adressierungs-Systeme (ATLAS) abgeleitet, die derzeit in einer Reihe von interdisziplinären Anwendungen erprobt wird. Solche Anwendungen betreffen beispielsweise:

- eine konstruktivistisch orientierte E-Learning-Umgebung für Unternehmensgründer,

- ein neuartiges Archivkonzept für die Musikwissenschaften und

- hochspezialisierte Kooperationsumgebungen für Menschen mit speziellen Behinderungen (Gehörlose, Aphasiker).

Insbesondere der letztgenannte Anwendungskreis zeigt, dass eine bewusste Steuerung von Transkriptionstechniken sowohl die Adressierbarkeit internetbasierter Medien deutlich verbessert als auch den Betroffenen wesentlich verbesserte Möglichkeiten zur medien-gestützten Selbstorganisation (Lokalisierung) bietet. Es deutet sich damit an, dass der zunächst medientheoretische Ansatz des Forschungskollegs auch praktische Bedeutung bei der Bewältigung einer der wichtigsten Herausforderungen an die Informatik der nächsten Jahre bieten kann: die Beherrschbarkeit des Mediums Internet (und des daraus resultierenden Ubiquitären Computing) nicht länger auf eine kleine Minderheit der Bevölkerung zu beschränken. 